Revista Bioética

\title{
PESQUISA
}

\section{Repercussões dos cuidados paliativos pediátricos: revisão integrativa}

Amanda Andrade Aguiar de Pinho ${ }^{1}$, Isabel Regiane Cardoso do Nascimento ${ }^{2}$, Igor Weyber da Silva Ramos ${ }^{3}$, Vanilla Oliveira Alencar ${ }^{4}$

1. Programa de Residência Médica em Neonatologia, Hospital Infantil Albert Sabin, Fortaleza/CE, Brasil. 2. Departamento de Psicologia, Hospital Infantil Albert Sabin, Fortaleza/CE, Brasil. 3. Departamento de Psicologia, Universidade de Fortaleza, Fortaleza/CE, Brasil. 4. Programa de Residência Multiprofissional em Saúde da Criança, Hospital Infantil Albert Sabin, Fortaleza/CE, Brasil.

\section{Resumo}

O artigo visa discutir os efeitos dos serviços de cuidados paliativos pediátricos sobre a instituição hospitalar e a qualidade de vida de pacientes e seus familiares. Para isso, o texto traz resultados de revisão integrativa de literatura que considerou 14 artigos publicados de 2013 a 2018. Nos estudos analisados sobressaíram os seguintes aspectos: eficácia dos cuidados paliativos pediátricos; diminuição dos custos hospitalares após a implantação do serviço; importância da equipe interdisciplinar; necessidades educacionais dos profissionais da saúde; e impacto na qualidade de vida de pacientes e familiares. Conclui-se que os cuidados paliativos pediátricos proporcionam cuidado transversal e devem ser reconhecidos como serviço especializado e indispensável para pacientes pediátricos com doenças incuráveis.

Palavras-chave: Cuidados paliativos. Pediatria. Serviços de saúde. Assistência hospitalar.

\section{Resumen}

\section{Repercusiones de los cuidados paliativos pediátricos: revisión integrativa}

Este artículo tiene como objetivo discutir los efectos de los servicios de cuidados paliativos pediátricos sobre la institución hospitalaria y la calidad de vida de los pacientes y su familia. Para ello, el texto presenta los resultados de una revisión integrativa de la literatura que incluyó 14 artículos publicados entre el 2013 y el 2018. En los estudios analizados destacaron los siguientes aspectos: la eficacia de los cuidados paliativos pediátricos, la reducción de los costos hospitalarios tras la implantación del servicio, la importancia del equipo interdisciplinar, las necesidades educativas de los profesionales de la salud; y el impacto en la calidad de vida de los pacientes y su familia. Se concluye que los cuidados paliativos pediátricos proporcionan cuidado transversal y deben ser reconocidos como un servicio especializado y esencial para los pacientes pediátricos con enfermedades incurables.

Palabras clave: Cuidados paliativos. Pediatría. Servicios de salud. Atención hospitalaria.

\section{Abstract \\ Repercussions of pediatric palliative care: an integrative review}

This article aims to discuss the effects of pediatric palliative care services on hospitals and the quality of life of patients and their families. The research reports the results of an integrative literature review based on 14 articles published between 2013 to 2018. In the studies analyzed, the following aspects were highlighted: efficacy of pediatrics palliative care in hospitals, reductions of hospital costs after implementation of the service, importance of multidisciplinary teams, educational needs of health professionals, and the impact on the quality of life of patients and their families. Results show that pediatrics palliative care provides comprehensive care and should be recognized as an indispensable and specialized service for children with incurable diseases.

Keywords: Palliative care. Pediatrics. Health services. Hospital care. 
Na segunda metade do século $X X$, progressos científicos e avanços terapêuticos tornaram possível realizar diagnósticos precoces. Entretanto, como efeito da maior expectativa de vida, doenças crônicas tornaram-se mais prevalentes. Essa tendência chama atenção para o sofrimento de pacientes com enfermidades incuráveis, trazendo novas demandas para os serviços de saúde e alterando o tipo de cuidado a ser prestado ao paciente e sua família.

Doenças que limitam ou ameaçam a vida atingem também crianças e, com elas, seus familiares. Trata-se muitas vezes de condições crônicas complexas, ou seja, situações médicas que tendem a durar pelo menos 12 meses e afetam gravemente sistemas e órgãos. Tais enfermidades exigem cuidados pediátricos especializados, com prováveis períodos de internação em serviços de atenção terciária, e são condições progressivas e sem possibilidade terapêutica de cura, inevitavelmente levando à morte ${ }^{1}$.

A Organização Mundial da Saúde (OMS) ${ }^{2}$ reconhece que cuidados paliativos promovem qualidade de vida ao prevenir e aliviar o sofrimento de pacientes acometidos por doenças graves com risco de morte. Esse tipo de assistência visa tratar a dor e outros sintomas, fornecendo também apoio psicossocial e espiritual, que se estende aos familiares ${ }^{2,3}$. A OMS $^{2}$ também considera os cuidados paliativos no contexto pediátrico, abrangendo suporte e orientação à família da criança.

Trata-se de assistência interdisciplinar que envolve cuidados totais, ativos e integrados, implementados quando a doença não responde a tratamentos curativos ${ }^{2,4}$. Os cuidados paliativos pediátricos (CPP) são iniciados quando a doença é diagnosticada, progredindo de acordo com a evolução do quadro clínico. É necessário avaliar individualmente cada criança e sua família, respeitando crenças e valores e facilitando a comunicação. A morte não encerra os CPP, que devem continuar durante o luto familiar ${ }^{5}$.

No contexto da pediatria, a atenção deve ser ainda maior, pois crianças são seres em desenvolvimento, que têm direito à devida proteção por parte do Estado, sobretudo quando doentes. Nesse contexto, torna-se essencial que os sistemas de saúde, em seus diversos níveis de complexidade, tenham equipes interprofissionais que ofertem CPP de modo integrado e com competência técnica. Hospitais de referência, principalmente, devem assimilar essa proposta e romper com os paradigmas centrados apenas na doença e em protocolos curativos. É necessário operacionalizar o início imediato dos cuidados paliativos após o diagnóstico da doença crônica, de forma concomitante ao tratamento curativo, que pode não ter efeito ${ }^{5}$.

Sobre o perfil clínico-epidemiológico dos pacientes que demandam CPP, observa-se na literatura prevalência de malformação congênita/genética, câncer, doenças neuromusculares, respiratórias e gastrointestinais ${ }^{5}$. A maioria desses pacientes depende de tecnologia médica, e a gastrostomia é o suporte mais comum, seguida de cateter de diálise, quimioterapia e traqueostomia ${ }^{5,6}$. Estudo de coorte destaca ainda que, em 12 meses de seguimento, $30,3 \%$ dos pacientes acabaram morrendo ${ }^{6}$.

A família do paciente, por sua vez, costuma demonstrar medo, culpa e ansiedade diante dos tratamentos e do futuro da criança, demandando atendimento especializado que responda a suas necessidades e forneça mais informações. Por isso, a atuação da equipe nos CPP deve ser interdisciplinar, com profissionais de diferentes áreas acompanhando a evolução e o prognóstico do paciente nas unidades de internação ou nas visitas domiciliares.

Todavia, como especialidade emergente, os CPP ainda estão em desenvolvimento. Muitos são os desafios para implantar esse tipo de assistência, como a heterogeneidade da população pediátrica, prognóstico incerto, carência de conhecimento, falta de suporte institucional e financeiro, vulnerabilidade do paciente e escassas pesquisas científicas sobre cuidados paliativos voltados a essa faixa etária ${ }^{7}$. Percebe-se ainda resistência em definir o prognóstico de terminalidade e foco da equipe médica em ações curativas, relegando a segundo plano o sofrimento biopsicossocial da criança ${ }^{8}$.

Diante da necessidade de implementar sistematicamente os CPP no âmbito hospitalar, este trabalho tem como objetivo discutir que fatores intervêm nesse processo e quais são as repercussões do cuidado paliativo na qualidade de vida de pacientes e familiares.

\section{Método}

Trata-se de estudo qualitativo de cunho exploratório que utilizou como procedimento técnico a revisão integrativa de literatura. Esse método tem por finalidade reunir e sintetizar resultados de pesquisas sobre determinado tema, de modo a permitir sua análise crítica e contribuir para a prática clínica ao aprofundar o conhecimento sobre a matéria investigada ${ }^{9}$. Foi realizada pesquisa nas bases de dados Literatura Latino-Americana e do Caribe em Ciências da Saúde (Lilacs) e Medical Literature Analysis 
and Retrieval System Online (Medline)/PubMed utilizando-se os descritores "cuidados paliativos" (palliative care), "pediatria" (pediatrics), "serviços de saúde" (health services) e "assistência hospitalar" (hospital care), em português e inglês.

Foram incluídos artigos em português ou inglês publicados na íntegra e indexados entre 2013 e 2018. Os textos podiam ter percursos metodológicos variados, mas deviam descrever a prestação dos cuidados paliativos em pediatria geral e as características dos pacientes atendidos, apresentando resultados conclusivos. Foram excluídos os artigos que se limitavam a abordar os cuidados paliativos em oncologia pediátrica, uma vez que nesse contexto a abordagem já está bem difundida, ou que tratavam do tema considerando doenças específicas. Publicações que não fossem artigos (como editoriais, cartas ao editor etc.) também foram desconsideradas.

A pesquisa inicial resultou em 615 artigos, cujos títulos e resumos foram lidos a fim de eliminar os textos que não correspondessem ao tema proposto. Desse processo restaram 60 artigos, lidos na íntegra. A partir dessa leitura, considerando os critérios de inclusão e exclusão, foram selecionados 14 artigos para compor a amostra.

\section{Resultados}

O Quadro 1 traz informações dos artigos selecionados para a pesquisa. São apresentados dados de autoria, ano de publicação, título, objetivos e base de dados em que o texto foi encontrado.

Quadro 1. Características da amostra

\begin{tabular}{|c|c|c|c|}
\hline Autores/ano & Título & Objetivos & Base de dados \\
\hline $\begin{array}{l}\text { Herbert e } \\
\text { colaboradores; } \\
2014^{10}\end{array}$ & \begin{tabular}{|l|} 
"Development of a state-wide \\
pediatric palliative care service in \\
Australia: referral and outcomes \\
over two years"
\end{tabular} & $\begin{array}{l}\text { Mapear as características da população atendida } \\
\text { pelo recém-formado serviço de cuidados paliativos } \\
\text { pediátricos em Queensland, Austrália, e descrever o } \\
\text { desenvolvimento do referido serviço por } 24 \text { meses. }\end{array}$ & $\begin{array}{l}\text { Medline/ } \\
\text { PubMed e } \\
\text { Lilacs }\end{array}$ \\
\hline $\begin{array}{l}\text { Verberne e } \\
\text { colaboradores; } \\
2018^{11}\end{array}$ & $\begin{array}{l}\text { "Barriers and facilitators to the } \\
\text { implementation of a paediatric } \\
\text { palliative care team" }\end{array}$ & $\begin{array}{l}\text { Identificar barreiras e facilitadores relatados } \\
\text { por profissionais de saúde em atenção primária, } \\
\text { secundária e terciária na implementação de serviço } \\
\text { de cuidados paliativos pediátricos que visa integrar } \\
\text { a assistência no hospital e no lar do paciente. }\end{array}$ & $\begin{array}{l}\text { Medline/ } \\
\text { PubMed }\end{array}$ \\
\hline $\begin{array}{l}\text { Verberne e } \\
\text { colaboradores; } \\
2017^{12}\end{array}$ & $\begin{array}{l}\text { "Parental experiences with a } \\
\text { paediatric palliative care team: a } \\
\text { qualitative study" }\end{array}$ & $\begin{array}{l}\text { Obter informações, com base na perspectiva dos } \\
\text { pais, sobre o suporte fornecido por nova equipe } \\
\text { pediátrica de cuidados paliativos. }\end{array}$ & $\begin{array}{l}\text { Medline/ } \\
\text { PubMed }\end{array}$ \\
\hline $\begin{array}{l}\text { Smith e } \\
\text { colaboradores; } \\
2015^{13}\end{array}$ & $\begin{array}{l}\text { "Pediatric palliative care and } \\
\text { inpatient hospital costs: a } \\
\text { longitudinal cohort study" }\end{array}$ & $\begin{array}{l}\text { Examinar a relação entre custo de internação e } \\
\text { cuidados paliativos pediátricos. }\end{array}$ & $\begin{array}{l}\text { Medline/ } \\
\text { PubMed e } \\
\text { Lilacs }\end{array}$ \\
\hline $\begin{array}{l}\text { Ananth e } \\
\text { colaboradores; } \\
2017^{14}\end{array}$ & $\begin{array}{l}\text { "Trends in hospital utilization and } \\
\text { costs among pediatric palliative } \\
\text { care recipients" }\end{array}$ & $\begin{array}{l}\text { Avaliar utilização hospitalar e custos comparando } \\
\text { pacientes que recebem cuidados paliativos } \\
\text { pediátricos com pacientes que não os recebem } \\
\text { no Boston Children's Hospital. }\end{array}$ & $\begin{array}{l}\text { Medline/ } \\
\text { PubMed }\end{array}$ \\
\hline $\begin{array}{l}\text { Conte e } \\
\text { colaboradores; } \\
2016^{15}\end{array}$ & $\begin{array}{l}\text { "Pediatric palliative care program } \\
\text { versus usual care and healthcare } \\
\text { resource utilization in British } \\
\text { Columbia: a matched-pairs } \\
\text { cohort study" }\end{array}$ & $\begin{array}{l}\text { Comparar uso de recursos hospitalares entre } \\
\text { crianças que recebem cuidados paliativos e } \\
\text { crianças sob cuidados habituais. }\end{array}$ & $\begin{array}{l}\text { Medline/ } \\
\text { PubMed }\end{array}$ \\
\hline $\begin{array}{l}\text { Gans e } \\
\text { colaboradores; } \\
2016^{16}\end{array}$ & $\begin{array}{l}\text { "Cost analysis and policy } \\
\text { implications of a pediatric } \\
\text { palliative care program" }\end{array}$ & $\begin{array}{l}\text { Descrever programa de cuidados paliativos } \\
\text { Partners for Children, criado na Califórnia, } \\
\text { abordando o contexto político do seu } \\
\text { desenvolvimento, e avaliar os efeitos da } \\
\text { iniciativa, considerando seu custo. }\end{array}$ & $\begin{array}{l}\text { Medline/ } \\
\text { PubMed }\end{array}$ \\
\hline $\begin{array}{l}\text { Jamorabo, } \\
\text { Belani, Martin; } \\
2015^{17}\end{array}$ & $\begin{array}{l}\text { "Complex chronic conditions } \\
\text { in Rhode Island's pediatric } \\
\text { populace: implications for } \\
\text { palliative and hospice services, } \\
\text { 2000-2012" }\end{array}$ & $\begin{array}{l}\text { Descrever as tendências de mortalidade de } \\
\text { crianças de até } 17 \text { anos de idade com condições } \\
\text { crônicas complexas em Rhode Island. }\end{array}$ & $\begin{array}{l}\text { Medline/ } \\
\text { PubMed e } \\
\text { Lilacs }\end{array}$ \\
\hline
\end{tabular}


Quadro 1. Continuação

\begin{tabular}{|c|c|c|c|}
\hline Autores/ano & Título & Objetivos & Base de dados \\
\hline $\begin{array}{l}\text { Ogelby, } \\
\text { Goldstein; } \\
2014^{18}\end{array}$ & $\begin{array}{l}\text { "Interdisciplinary care: using } \\
\text { your team" }\end{array}$ & $\begin{array}{l}\text { Revisar a estrutura atual da equipe de cuidados } \\
\text { paliativos, mostrando oportunidades de incluir } \\
\text { outros membros e identificando desafios } \\
\text { que surgem quando se amplia o grupo de } \\
\text { profissionais envolvidos no cuidado de uma } \\
\text { criança e de sua família. }\end{array}$ & $\begin{array}{l}\text { Medline/ } \\
\text { PubMed }\end{array}$ \\
\hline $\begin{array}{l}\text { Moore, Sheetz; } \\
2014^{19}\end{array}$ & $\begin{array}{l}\text { "Pediatric palliative care } \\
\text { consultation" }\end{array}$ & $\begin{array}{l}\text { Refletir sobre os benefícios do alinhamento entre } \\
\text { equipe de saúde e paciente e família, partindo } \\
\text { das consultas em cuidados paliativos. }\end{array}$ & $\begin{array}{l}\text { Medline/ } \\
\text { PubMed e } \\
\text { Lilacs }\end{array}$ \\
\hline $\begin{array}{l}\text { Keele, Keenan, } \\
\text { Bratton; } 2016^{20}\end{array}$ & $\begin{array}{l}\text { "The effect of palliative care } \\
\text { team design on referrals to } \\
\text { pediatric palliative care" }\end{array}$ & $\begin{array}{l}\text { Descrever a estrutura da equipe de cuidados } \\
\text { paliativos, avaliando se a composição e a } \\
\text { disponibilidade estão associadas à utilidade, e } \\
\text { examinar padrões de referência. }\end{array}$ & $\begin{array}{l}\text { Medline/ } \\
\text { PubMed }\end{array}$ \\
\hline $\begin{array}{l}\text { Frizzola, Miller; } \\
2014^{21}\end{array}$ & $\begin{array}{l}\text { "Referrals to a new pediatric } \\
\text { palliative care team: details of } \\
\text { the first } 12 \text { months of operation" }\end{array}$ & $\begin{array}{l}\text { Descrever a população de pacientes assistida por } \\
\text { programa de cuidados paliativos recém-criado, } \\
\text { comparando-a com a literatura existente. }\end{array}$ & $\begin{array}{l}\text { Medline/ } \\
\text { PubMed e } \\
\text { Lilacs }\end{array}$ \\
\hline $\begin{array}{l}\text { Benini e } \\
\text { colaboradores; } \\
2016^{22}\end{array}$ & $\begin{array}{l}\text { "Barriers to the development of } \\
\text { pediatric palliative care in Italy" }\end{array}$ & $\begin{array}{l}\text { Destacar, mediante revisão de dados e da } \\
\text { literatura, questões críticas que impedem o } \\
\text { planejamento e o desenvolvimento do serviço de } \\
\text { cuidados paliativos na Itália. }\end{array}$ & $\begin{array}{l}\text { Medline/ } \\
\text { PubMed }\end{array}$ \\
\hline $\begin{array}{l}\text { Goldhagen e } \\
\text { colaboradores; } \\
2016^{23}\end{array}$ & $\begin{array}{l}\text { "Community-based pediatric } \\
\text { palliative care for health related } \\
\text { quality of life, hospital utilization } \\
\text { and costs lessons learned from a } \\
\text { pilot study" }\end{array}$ & $\begin{array}{l}\text { Analisar resultados de estudos de avaliação não } \\
\text { publicados, realizados a partir de } 2007 \text {, que } \\
\text { demonstram o impacto do programa de cuidados } \\
\text { paliativos comunitários da PedsCare na qualidade de } \\
\text { vida de pacientes e na utilização e custo hospitalar. }\end{array}$ & $\begin{array}{l}\text { Medline/ } \\
\text { PubMed }\end{array}$ \\
\hline
\end{tabular}

\section{Caracterização dos dados}

Quatro artigos que compõem a amostra foram publicados em $2014^{10,18,19,21}$, dois em $2015^{13,17}$, cinco em 2016 ${ }^{15,16,20,22,23}$, dois em $2017^{12,14}$ e um em $2018^{11}$. Todos os artigos identificados foram produzidos fora do Brasil. Seis deles foram publicados no Journal of Palliative Medicine ${ }^{10,14,15,17,20,21}$, dois em Pediatric Clinics of North America ${ }^{18,19}$, dois em BMC Palliative Care $^{11,23}$ e os demais em Palliative Medicine ${ }^{12}$, Annali dell'Istituto Superiore di Sanità ${ }^{22}$, Journal of Pain and Symptom Management ${ }^{16}$ e Pediatrics ${ }^{13}$.

Quanto ao método, um artigo foi revisão de literatura ${ }^{23}$. O delineamento qualitativo foi visto em dois trabalhos, com uso de entrevistas ${ }^{12}$ ou questionários ${ }^{10}$. Outros nove trabalhos ${ }^{11,13-16,18,19,21,22}$ têm abordagem quantitativa, com predomínio de estudos de coorte. Em um estudo ${ }^{17}$ houve análise qualitativa (por entrevista) e quantitativa (por análise de custos) e outro teve caráter descritivo ${ }^{20}$.

A literatura pesquisada apresentou fatores que se sobressaíram e serão discutidos individualmente: a eficácia dos cuidados paliativos em hospitais; a diminuição dos custos hospitalares com a implantação desse tipo de assistência; a importância da equipe interdisciplinar; as necessidades educacionais dos profissionais de saúde; e o impacto na qualidade de vida de pacientes e familiares.

\section{Discussão}

\section{Eficácia dos cuidados paliativos}

Herbert e colaboradores ${ }^{10}$ tratam da implementação dos CPP no estado de Queensland, Austrália, onde essa assistência é definida como serviço consultivo e interdisciplinar que expande o cuidado para além do hospital, independentemente do diagnóstico e do local em que reside a criança, por meio de visita domiciliar, videoconferência ou telessaúde. Paciente e familiares são assistidos desde o encaminhamento ao serviço referenciado e o diagnóstico, continuando mesmo após a alta hospitalar. Além de prestar serviços de consulta para crianças internadas e oferecer ambulatórios especializados nos hospitais, o estado também fornece assistência direta, em conjunto com outros serviços comunitários.

Ainda que o estudo aborde dificuldades de provar a eficácia do serviço e mensurar a experiência individual dos pacientes, os depoimentos 
apresentados por familiares e pelas equipes de saúde demonstram visão positiva dos cuidados paliativos. Os relatos consideram o serviço valioso e eficaz, capaz de atender às necessidades da comunidade. Destacam-se a coordenação do cuidado e a promoção da qualidade de vida de pacientes e familiares, que se estendem para além do ambiente hospitalar ${ }^{10}$.

Verberne e colaboradores ${ }^{11}$, por sua vez, avaliaram a percepção de profissionais da atenção primária, secundária e terciária sobre a equipe de cuidados paliativos, considerados relevantes para a criança e sua família. Essa percepção motiva os profissionais a colaborar com os CPP, que não substituem a assistência clínica regular, mas coordenam o cuidado em torno da criança e da família. O objetivo é fornecer atenção baseada na confiança, compartilhando informações e os mesmos objetivos ${ }^{11}$.

Em outro estudo, avaliando a percepção de pais de pacientes, Verbene e colaboradores ${ }^{12}$ demonstram que famílias apresentam expectativas limitadas quanto à equipe de cuidados paliativos. Muitos pais têm dificuldade em aceitar a necessidade desses cuidados para o filho. Todavia, a tendência é que aos poucos a equipe ganhe confiança da família e tenha seu trabalho valorizado, destacando-se os seguintes aspectos: continuidade e coordenação dos cuidados, estabelecimento de ponto de contato, suporte prático e atitude sensível e confiável dos profissionais. Ressalta-se a prática das visitas domiciliares e, como ponto de melhoria, os pais sugerem esclarecimentos antecipados sobre o trabalho da equipe ${ }^{12}$.

Diante do exposto, percebe-se que os cuidados paliativos possibilitam atenção transversal, pautada nos preceitos de humanização e assistência hospitalar interdisciplinar - princípios que regem os serviços de saúde.

\section{Diminuição de custos hospitalares}

Smith e colaboradores ${ }^{13}$ observaram que os custos financeiros da internação de crianças que receberam cuidados paliativos variam em relação aos daquelas que não os receberam. Os autores reconhecem que se trata de inter-relação bastante complexa, mas os resultados sugerem menor custo nos casos em que esses serviços foram implementados. O tempo de internação também foi menor, o que demonstra a importância dos cuidados pré-hospitalares e da priorização da qualidade de vida. Além disso, os cuidados paliativos aumentam a satisfação dos familiares assistidos pela equipe.
O trabalho tem como principais limitações a inclusão de apenas pacientes de alto custo e o uso de dados administrativos de uma única instituição ${ }^{13}$.

Ananth e colaboradores ${ }^{14}$ também demonstraram que pacientes com doenças graves que receberam cuidados paliativos tiveram menos internações hospitalares e visitaram menos a emergência. Apesar da tendência de aumento de admissões na unidade de terapia intensiva após o início do cuidado paliativo, no hospital estudado os custos permaneceram estáveis, possivelmente em razão de reduções no uso de recursos hospitalares, ainda que ventilação mecânica e assistência tecnológica tenham sido empregados na mesma medida ${ }^{14}$.

Pode haver mudanças sutis no uso de recursos, como a redução de abordagens cirúrgicas observada por Ananth e colaboradores ${ }^{14}$. No entanto, para pacientes terminais, os cuidados paliativos podem não alterar substancialmente o uso hospitalar - dado encontrado também por Smith e colaboradores ${ }^{13}$ e Conte e colaboradores ${ }^{15}$. Embora os mecanismos exatos do decréscimo no uso hospitalar requeiram mais investigação, pode-se afirmar que os CPP facilitam a realocação de cuidados hospitalares para o nível ambulatorial, com conversas antecipadas sobre planejamento de cuidados incluindo equipe assistencial, paciente e familiares.

Em seu trabalho, Gans e colaboradores ${ }^{16}$ apresentam o programa de CPP da Califórnia, que oferece apoio domiciliar, reduzindo custos e internações hospitalares por meio de cuidados comunitários. Calcula-se economia de US\$ 3.331 mensais por paciente, além de redução de quase $50 \%$ na média de internações mensais. Esse importante resultado foi viabilizado pela diminuição da frequência e duração das hospitalizações, com aumento relativamente menor de custos ambulatoriais. O tempo de internação foi de em média 16,7 dias antes da inscrição no programa de cuidados paliativos para 6,5 dias após a inscrição ${ }^{16}$.

Diante dos poucos estudos que abordam a redução de custos decorrente dos cuidados paliativos e da dificuldade de analisar informações financeiras de hospitais, percebe-se que a temática ainda precisa ser pesquisada de modo mais aprofundado.

\section{Importância da equipe interdisciplinar}

No estudo de Jamorabo, Belani e Martin ${ }^{17}$, as condições crônicas complexas são apontadas como a principal causa de morte de crianças de 1 a 17 anos. Condições perinatais e anormalidades genéticas e congênitas podem afetar vários órgãos 
e produzir outras comorbidades. A diversidade dessas condições reforça a necessidade da abordagem interdisciplinar, ajudando as famílias a lidar com possíveis complicações e a adaptar o domicílio de forma apropriada ${ }^{17}$.

A pesquisa também evidenciou que a maioria dos óbitos ocorre em unidades de emergência ou no domicílio, enquanto pequeno número se dá em instituição de cuidados paliativos, o que reflete a escassez desse tipo de serviço ${ }^{17}$. Entretanto, pode-se apontar como limitação desse estudo de coorte a documentação insuficiente para esclarecer a oferta de CPP e os tipos de intervenção realizados. A pesquisa partiu de inferências baseadas em atestados de óbito ${ }^{17}$.

Importante característica das equipes de cuidados paliativos é que diferentes membros podem assumir a liderança, a depender dos objetivos da família e das necessidades de dado momento. Ogelby e Goldstein ${ }^{18}$ demostram a natureza flexível dessas equipes, que se distinguem do modelo de medicina tradicional - no qual médicos ocupam o topo da hierarquia, centralizando as decisões. O cuidado interdisciplinar estimula a comunicação contínua entre a equipe, de modo a reduzir o ônus da família em ter que coordenar os cuidados e transmitir informações aos mais diversos profissionais. Com abordagem claramente unificada, as deliberações têm maior probabilidade de serem resolvidas em comum acordo, e é menos provável que a tomada de decisões delicadas seja prejudicada pela opinião de um cuidador descoordenado do restante da equipe ${ }^{18}$.

Moore e Sheetz ${ }^{19}$ apontam que em consultas de cuidados paliativos se visa mais frequentemente manejar sintomas, facilitar a comunicação, tomar decisões, auxiliar a coordenação de cuidados e a transição para casa e discutir ordens de não ressuscitar. Os autores reforçam que o cuidado das crianças e de sua família deve ser coordenado por equipe interdisciplinar, e que é necessário integrar os diversos níveis de atenção da assistência (primária, secundária e terciária) ${ }^{19}$.

É preciso que hospitais sejam capazes de organizar equipes de cuidados paliativos com membros adequados, mas não devem retardar sua composição à espera de melhores recursos. Como defendem Keele, Keenan e Bratton ${ }^{20}$, as instituições devem ser incentivadas a iniciar os cuidados paliativos com os meios disponíveis, contratando os profissionais que lhes faltam no decorrer do tempo - o que pode inclusive aumentar o número de encaminhamentos ${ }^{20}$.
Frizzola e Miller ${ }^{21}$, por sua vez, discutem a implantação do serviço de cuidados paliativos em hospital infantil, funcionando já no primeiro ano com demanda maior que a esperada. A rápida aceitação foi creditada ao bom planejamento, à liderança do hospital e ao forte apoio clínico, administrativo e financeiro da instituição. Houve ainda grande esforço educacional antes do início do programa, promovendo mudança cultural que favoreceu o acolhimento dos cuidados paliativos em hospitais pediátricos. O cenário contrasta com o período de estagnação inicial previsto pela literatura e mencionado pelas próprias autoras ${ }^{21}$.

\section{Educação dos profissionais}

Benini e colaboradores ${ }^{22}$ indicam como grande desafio para formar equipes de cuidados paliativos a falta de treinamento nesse tipo de assistência nos currículos acadêmicos. A formação da equipe depende da disponibilidade de profissionais de saúde com conhecimento e experiência específicos na prestação desses cuidados. Contudo, em geral, o conhecimento dos profissionais nessa área é incompleto ou inadequado, impedindo resposta qualificada e equitativa às necessidades da população ${ }^{22}$.

Além de conhecimento e experiência clínica, membros de equipes de cuidados paliativos devem ter capacidade de organização, espírito de equipe, habilidades de comunicação e relacionamento interpessoal, e ainda conseguir compreender dilemas éticos ${ }^{22}$. A ausência dessas habilidades prejudica a relação entre médicos e familiares dos pacientes, entre profissionais com diferentes especializações, entre membros da equipe e até entre pacientes e seus familiares. A falta de profissionais adequadamente treinados é, portanto, a principal causa da resposta limitada ou ineficaz das crianças ao cuidado paliativo, afetando inclusive a credibilidade desse tipo de atenção ${ }^{22}$.

\section{Qualidade de vida de pacientes e seus familiares}

Benini e colaboradores ${ }^{22}$ abordam ainda as consequências do diagnóstico de doença incurável em uma criança. É grande a dificuldade de tratar as necessidades do paciente, o sofrimento da família e o ônus do cuidado ${ }^{22}$, e o impacto do diagnóstico prejudica a qualidade de vida tanto da criança como de seus familiares, cuja rotina é severamente modificada pelo tratamento prolongado em ambiente hospitalar. Adaptações especiais se fazem necessárias - incluindo aquelas voltadas à atividade escolar do paciente e à atividade 
profissional dos cuidadores -, o que muitas vezes implica na descontinuidade dos projetos de todos os envolvidos. Os pais muitas vezes experimentam sentimentos intensos - impotência, frustração, medo e ansiedade - que podem interferir na capacidade de tomar decisões ${ }^{22}$.

O trabalho de Benini e colaboradores ${ }^{22}$ sugere que a relutância em desistir é fator determinante dos cuidados escolhidos pelos pais para seus filhos. Nesse contexto, a incapacidade de aceitar a impossibilidade de cura e a convicção de que o encaminhamento para cuidados paliativos equivale a desistir são as questões mais críticas ${ }^{22}$. A comunicação honesta e detalhada é fundamental para ajudar os familiares a aceitar o prognóstico da criança e prepará-los para as necessidades presentes e futuras do paciente. É preciso esclarecer os pais de que os cuidados paliativos são considerados o meio mais apropriado para ajudar crianças com doenças graves a alcançar a melhor qualidade de vida possível, protegendo sua dignidade e a de sua família ${ }^{22}$.

\section{Considerações finais}

Cuidados paliativos proporcionam assistência transversal nos diversos níveis de atenção por meio de equipes preparadas para atuar de forma interdisciplinar, apoiando os familiares e melhorando a qualidade de vida do paciente ao Ihe garantir mais autonomia no processo de saúde-doença e fim de vida. Sugere-se que sejam realizados estudos que apresentem experiências brasileiras e apontem dificuldades em comum, visando melhorar serviços com realidades semelhantes. Também se percebe a necessidade de pesquisas que abordem a perspectiva da criança sobre os diversos assuntos relacionados à qualidade de vida e ao adoecimento, a fim de melhorar o cuidado prestado.

Este estudo buscou dar um passo nesse sentido ao fornecer informações para a implantação de serviços de cuidados paliativos no país, os quais humanizam e integralizam a assistência à saúde, e por isso devem ser reconhecidos como serviço especializado e indispensável para pacientes pediátricos com doenças incuráveis.

Artigo originário do trabalho de conclusão de curso da residência médica em pediatria geral pelo Hospital Albert Sabin, Fortaleza/CE, Brasil.

\section{Referências}

1. Lacerda AF, coordenadora. Cuidados paliativos pediátricos: relatório do Grupo de Trabalho do Gabinete do Secretário de Estado Adjunto do Ministro da Saúde [Internet]. Lisboa: GTGSEAMS; 2014 [acesso 8 jan 2019]. DOI: 10400.4/1871

2. World Health Organization. Palliative care [Internet]. 2019 [acesso 8 jan 2019]. Disponível: https://bit.ly/2HruiLU

3. Sociedade Brasileira de Pediatria. Departamento Científico de Medicina da Dor e Cuidados Paliativos. Cuidados paliativos pediátricos: o que são e qual sua importância? Cuidando da criança em todos os momentos [Internet]. São Paulo: Sociedade Brasileira de Pediatria; 2017 [acesso 8 jan 2019]. Disponível: https://bit.ly/2HrUoOU

4. Andrade CG, Alves AMPM, Costa SFG, Santos FS. Cuidados paliativos ao paciente em fase terminal. Rev Baiana Enferm [Internet]. 2017 [acesso 8 jan 2019];28(2):126-33. DOI: 10.18471/rbe.v28i2.9034

5. Valadares MTM, Mota JAC, Oliveira BM. Cuidados paliativos em pediatria: uma revisão. Rev. bioét (Impr.) [Internet]. 2013 [acesso 19 jan 2019];21(3):486-93. DOI: 10.1590/S1983-80422013000300013

6. Feudtner C, Kang TI, Hexem KR, Friedrichsdorf SJ, Osenga K, Siden $\mathrm{H}$ et al. Pediatric palliative care patients: a prospective multicenter cohort study. Pediatrics [Internet]. 2011 [acesso 8 jan 2019];127(6):94-101. DOI: 10.1542/peds.2010-3225

7. Schinzari NRG, Santos FS. Assistência à criança em cuidados paliativos na produção científica brasileira. Rev Paul Pediatr [Internet]. 2014 [acesso 8 jan 2019];32(1):99-106. DOI: 10.1590/S010305822014000100016

8. Floriani CA. Cuidados paliativos no domicílio: desafios aos cuidados de crianças dependentes de tecnologia. J Pediatr [Internet]. 2010 [acesso 26 jan 2019];86(1):15-20. DOI: 10.1590/S002175572010000100004

9. Mendes KDS, Silveira RCCP, Galvão CM. Revisão integrativa: método de pesquisa para a incorporação de evidências na saúde e na enfermagem. Texto Contexto Enferm [Internet]. 2008 [acesso 26 jan 2019];17(4):758-64. DOI: 10.1590/S0104-07072008000400018

10. Herbert A, Bradford N, Donovan L, Pedersen LA, Irving H. Development of a state-wide pediatric palliative care service in Australia: referral and outcomes over two years. J Palliat Med [Internet]. 2014 [acesso 26 jan 2019];17(3):288-95. DOI: 10.1089/jpm.2013.0400

11. Verberne LM, Kars MC, Schepers SA, Schouten-van Meeteren AYNS, Grootenhuis MA, van Delden $\mathrm{JJM}$. Barriers and facilitators to the implementation of a paediatric palliative care team. BMC Palliat Care [Internet]. 2018 [acesso 26 jan 2019];17:23. DOI: 10.1186/s12904-018-0274-8 
12. Verberne LM, Schouten-van Meeteren AY, Bosman DK, Colenbrander DA, Jagt CT, Grootenhuis MA et al. Parental experiences with a paediatric palliative care team: a qualitative study. Palliat Med [Internet]. 2017 [acesso 26 jan 2019];31(10):956-63. DOI: 10.1177/0269216317692682

13. Smith AG, Andrews S, Bratton SL, Sheetz J, Feudtner C, Zhong W et al. Pediatric palliative care and inpatient hospital costs: a longitudinal cohort study. Pediatrics [Internet]. 2015 [acesso 8 jan 2019];135(4):694-700. DOI: 10.1542/peds.2014-3161

14. Ananth P, Melvin P, Berry JG, Wolfe J. Trends in hospital utilization and costs among pediatric palliative care recipients. J Palliat Med [Internet]. 2017 [acesso 8 jan 2019];20(9):946-53. DOI: 10.1089/jpm.2016.0496

15. Conte T, Mitton C, Erdelyi S, Chavoshi N, Siden H. Pediatric palliative care program versus usual care and healthcare resource utilization in British Columbia: a matched-pairs cohort study. J Palliat Med [Internet]. 2016 [acesso 15 jan 2019];19(11):1218-23. DOI: 10.1089/jpm.2016.0177

16. Gans D, Hadler MW, Chen X, Wu S-H, Dimand R, Abramson JM et al. Cost analysis and policy implications of a pediatric palliative care program. J Pain Symptom Manage [Internet]. 2016 [acesso 17 jan 2019];52(3):329-35. DOI: 10.1016/j.jpainsymman.2016.02.020

17. Jamorabo DS, Belani CP, Martin EW. Complex chronic conditions in Rhode Island's pediatric populace: implications for palliative and hospice services, 2000-2012. J Palliat Med [Internet]. 2015 [acesso 14 jan 2019];18(4):350-7. DOI: 10.1089/jpm.2014.0226

18. Ogelby M, Goldstein RD. Interdisciplinary care: using your team. Pediatr Clin North Am [Internet]. 2014 [acesso 17 jan 2019];61(4):823-34. DOI: 10.1016/j.pcl.2014.04.009

19. Moore D, Sheetz J. Pediatric palliative care consultation. Pediatr Clin North Am [Internet]. 2014 [acesso 14 jan 2019];61(4):735-47. DOI: 10.1016/j.pcl.2014.04.007

20. Keele L, Keenan HT, Bratton SL. The effect of palliative care team design on referrals to pediatric palliative care. J Palliat Med [Internet]. 2016 [acesso 14 jan 2019];19(3):286-91. DOI: $10.1089 / \mathrm{jpm} .2015 .0261$

21. Frizzola M, Miller EG. Referrals to a new pediatric palliative care team: details of the first 12 months of operation. J Palliat Med [Internet]. 2014 [acesso 20 jan 2019];17(5):585-8. DOI: 10.1089/ jpm.2013.0206

22. Benini F, Orzalesi M, Santi A, Congedi S, Lazzarin P, Pellegatta F et al. Barriers to the development of pediatric palliative care in Italy. Ann Ist Super Sanità [Internet]. 2016 [acesso 19 jan 2019];52(4):558-64. DOI: 10.4415/ANN_16_04_16

23. Goldhagen J, Fafard M, Komatz K, Eason T, Livingood WC. Community-based pediatric palliative care for health related quality of life, hospital utilization and costs lessons learned from a pilot study. BMC Palliat Care [Internet]. 2016 [acesso 15 jan 2019];15(1):73. DOI: 10.1186/s12904-016-0138-z

\section{Participação dos autores}

Os autores participaram igualmente na elaboração do artigo.

\section{Correspondência}

Amanda Andrade Aguiar de Pinho - Rua Bonfim Sobrinho, 540, apt. 701 A, Fátima CEP 60040-500. Fortaleza/CE, Brasil.

Amanda Andrade Aguiar de Pinho - Especialista - amandaandaguiar@hotmail.com

\section{(iD) 0000-0003-2885-5070}

Isabel Regiane Cardoso do Nascimento - Mestre - isabel.cardoso@hias.ce.gov.br

(iD) 0000-0002-5665-0577

Igor Weyber da Silva Ramos - Graduado - igorweyber10@gmail.com

(iD) 0000-0002-2788-4219

Vanilla Oliveira Alencar - Graduada - vanilla.oliveira@hotmail.com

(iD) $0000-0002-4269-7147$

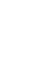

. 